\title{
Comparison of Semivariogram Models in Rain Gauge Network Design
}

\author{
${ }^{1}$ Mohd Khairul Bazli Mohd Aziz*, ${ }^{2}$ Fadhilah Yusof, ${ }^{3}$ Zalina Mohd Daud, ${ }^{4}$ Zulkifli Yusop \\ and ${ }^{5}$ Mohammad Afif Kasno \\ ${ }^{1}$ Faculty of Industrial Sciences \& Technology, Universiti Malaysia Pahang \\ 26300 Gambang, Pahang, Malaysia \\ ${ }^{2}$ Department of Mathematical Sciences, Faculty of Science \\ Universiti Teknologi Malaysia, 81310 Johor Bahru, Malaysia \\ ${ }^{3}$ UTM Razak School of Engineering and Advanced Technology \\ Universiti Teknologi Malaysia, Malaysia \\ ${ }^{4}$ Institute of Environmental and Water Resource Management (IPASA) \\ Faculty of Civil Engineering, Universiti Teknologi Malaysia, Malaysia \\ ${ }^{5}$ Department of Electronic \& Computer Engineering Faculty \\ Universiti Teknikal Malaysia, Melaka, Malaysia \\ ${ }^{*}$ Corresponding author: khairulbazli@ump.edu.my
}

Article history

Received: 4 October 2018

Received in revised form: 6 March 2019

Accepted: 14 May 2019

Published online: 1 August 2019

\begin{abstract}
The well-known geostatistics method (variance-reduction method) is commonly used to determine the optimal rain gauge network. The main problem in geostatistics method to determine the best semivariogram model in order to be used in estimating the variance. An optimal choice of the semivariogram model is an important point for a good data evaluation process. Three different semivariogram models which are Spherical, Gaussian and Exponential are used and their performances are compared in this study. Cross validation technique is applied to compute the errors of the semivariograms. Rainfall data for the period of 1975 - 2008 from the existing 84 rain gauge stations covering the state of Johor are used in this study. The result shows that the exponential model is the best semivariogram model and chosen to determine the optimal number and location of rain gauge station.
\end{abstract}

Keywords Geostatistics; semivariogram; rain gauge.

Mathematics Subject Classification 62P12, 86A32

\section{Introduction}

A hydrological network is an organized system for the collection of specific data such as precipitation, water quality, rainfall, streamflow and other climate parameters. The accuracy in the decision making of a water project design such as flood prediction depends on how much 
information is available for the area concerned. This is because sufficient accurate hydrologic data reduces the possibility of overdesign and thus minimizes the economic losses. There are several ways to define the objectives of the rain gauges network design, but the fundamental one in most studies is the selection of the optimum number of rain gauges stations and their optimum locations. The main objective of providing an optimal network of rain gauges is to adequately sample the rainfall and explain its nature of variability within the region concern. The rainfall changeability depends on wind, topography, the movement of storm and the type of storm. The location and spacing of gauges are also depending on the mentioned factors. Networks are often designed to monitor rainfall for resource assessment, design, operations and flood warning schemes.

Earlier studies on meteorological network design and optimization showed that variance reduction method of geostatistical method as one of the most popular method adopted by researchers. Bras and Iturbe [1] recognized rainfall as multidimensional stochastic process. By using the knowledge of such process and multivariate estimation theory, they developed a procedure for designing an optimal network to obtain the areal mean precipitation of an event over a fixed area. The methodology used in this research considered three different aspects of network design; spatial uncertainty and correlation of process, errors in measurement techniques and their correlation and nonhomogeneous sampling costs. The optimization technique used in this research is a search moving in the direction of highest partial gradient. They found out that the optimal networks (number and locations of rain gauges) will optimize the resulting cost and mean square error of rainfall estimation.

Bastin et al. [2] meanwhile, modeled the rainfall as two-dimensional random field. They proposed a simple procedure for the real-time estimation of the average rainfall over a catchment area using linear unbiased variance estimation method (kriging). They implement the method in two river basins in Belgium and the result showed that the method can be used for optimal selection of rain gauge location in a basin. Shamsi et al. [3] on the other hand, applied Universal kriging techniques based on the generalized covariances corresponding to IRF-k theory to analyze the design of rain gauge networks in regions where the spatial mean is not constant. Symmetric and asymmetric hypothetical rainfall fields were considered in order to obtain an optimal estimation of the watershed precipitation. The result showed that kriging method took into account the spatial variability of the storm within the catchment and not only the location of the rain gauges.

Kassim and Kottegoda [4] used simple and disjunctive kriging method and compared the estimation of optimum locations of recording rain gauges as part of a network for the determination of storm characteristics to be used in forecasting and design. The method was applied in the area of the Severn-Trent water basin, UK. Loof et al. [5] introduced the concept of 'regionalized variables' and the theory of kriging. They developed a methodology for selecting the best locations from a given number of rain gauges planned to be added in a network that is based on the spatial variability of the precipitation obtained by kriging. The methodology was applied in Karnali river basin, Nepal. The result showed that kriging can be of valuable use in identifying the optimal locations for a set of additional rain gauges using kriging standard deviation as an indicator.

Pardo [6] presented a method for establishing an optimal network design for the estimation of areal averages of rainfall events. In his study, he combined geostatistical variance-reduction method with simulated annealing as an algorithm of minimization. Chen et al. [7] proposed a 
method composed of kriging and entropy that can determine the optimum number and spatial distribution of rain gauge stations in catchments. The method was applied in Shimen Reservoir, Taiwan. The result showed that only seven rain gauge stations were needed to provide the necessary information.

Cudennec et al. [8] compared three differents geostatistical algorithms such as kriging with external drift, regression-kriging and cokriging to predict rainfall maps. The estimation variance was used to locate the regions where new stations must be added to obtain less important error estimation. This algorithm has been utilized in Tunisia. Chebbi et al. [9] proposed a method for assessing the optimal location of new monitoring stations within an existing rain gauge network. It took into account of precipitation as well as the prediction accuracy of rainfall erosivity. They used variance-reduction method with simulated annealing as an algorithm for objective function minimization to define the optimal network in Tunisia.

Awadallah [10] determined the spatial distribution of potential rainfall gauging stations by using the methodology based on the sequential use of kriging and entropy principles. Kriging is used to compute the spatial variations of rainfall in the locations of candidate stations. The candidate stations are prioritized using the entropy information. The methodology is applied on Makkah watershed.

Al-Aboodi and Al-Abadi [11] in 2014 used ordinary kriging technique to design optimum rain gauges network in Iraq. A new optimum network is designed by the trial and error procedure via add stations to the zones with higher standard error or drop or relocate in the zone of low standard error. Results indicate that 17 stations are required to optimize rain-gauges network with allowable error of about 10\%. Adhikary et al. [12] in 2014 design optimal rain gauge network in Middle Yarra, Australia by using kriging approach. Reduction of kriging error was considered as an indicator to optimally relocating of existing redundant stations.

Since then a lot of progress has been made in the development of geostatistics techniques of data analysis and interpolation. For this reason, geostatistics has become an extremely powerful tool for studying and evaluating space and/or time-related phenomena, and in the present days, its own techniques are implemented in all of the most popular data analysis softwares. Presently, geostatistics supplies a collection of powerful techniques that address the study of spatial correlation between experimental values of a specific variable in order to estimate values in unknown points inside the phenomenon existing domain. The basic tool of geostatistics and kriging is the semivariogram. The semivariogram captures the spatial dependence between samples by plotting semivariance against separation distance. For the kriging purpose, the empirical semivariogram must be replaced with an acceptable semivariogram model. This is because kriging algorithm will need access to semivariogram values for lag distances other than those used in the empirical semivariogram. More importantly, the semivariogram models used in the kriging process need to obey certain numerical properties in order for the kriging equations to be solvable. (Technically, the semivariogram model needs to be non-negative definite, in order the system of kriging equations to be non-singular.) Therefore, geostatisticians choose from a palette of acceptable or licit semivariogram models [13]. An optimal choice of the semivariogram model is an important point for a good data evaluation process. Since semivariogram expresses the relationship between measured values themselves, it is obvious that the model recognition strongly influences all the evaluation process [14]. Therefore, the performance of the Gaussian, exponential and spherical semivariogram model was compared in this study. 


\section{Study Area}

Johor is the second largest state in the Malaysia Peninsular, with an area of $18,941 \mathrm{~km}^{2}$. The rivers in Johor and its streams are important sources of water supply for the people of Johor. The catchment area contains a dense rain gauge network, 84 rain gauges covering 19,210 $\mathrm{km}^{2}$ in Johor (see Figure 1). The data used in this study are daily rainfall, from November 1975 until February 2008. The data was obtained from Department of Irrigation and Drainage (DID) Malaysia and Malaysia Meteorological Department (MMD). As shown in Figure 1, it is noticeable that there is no rain gauge station located along the Titiwangsa Mountains. This is because the rain gauge station cannot be placed in a hilly area because of the wind effect. The reason for this is because the increment of the height results to increment of the wind which influences the increment of error associated with the measured rainfall [15].

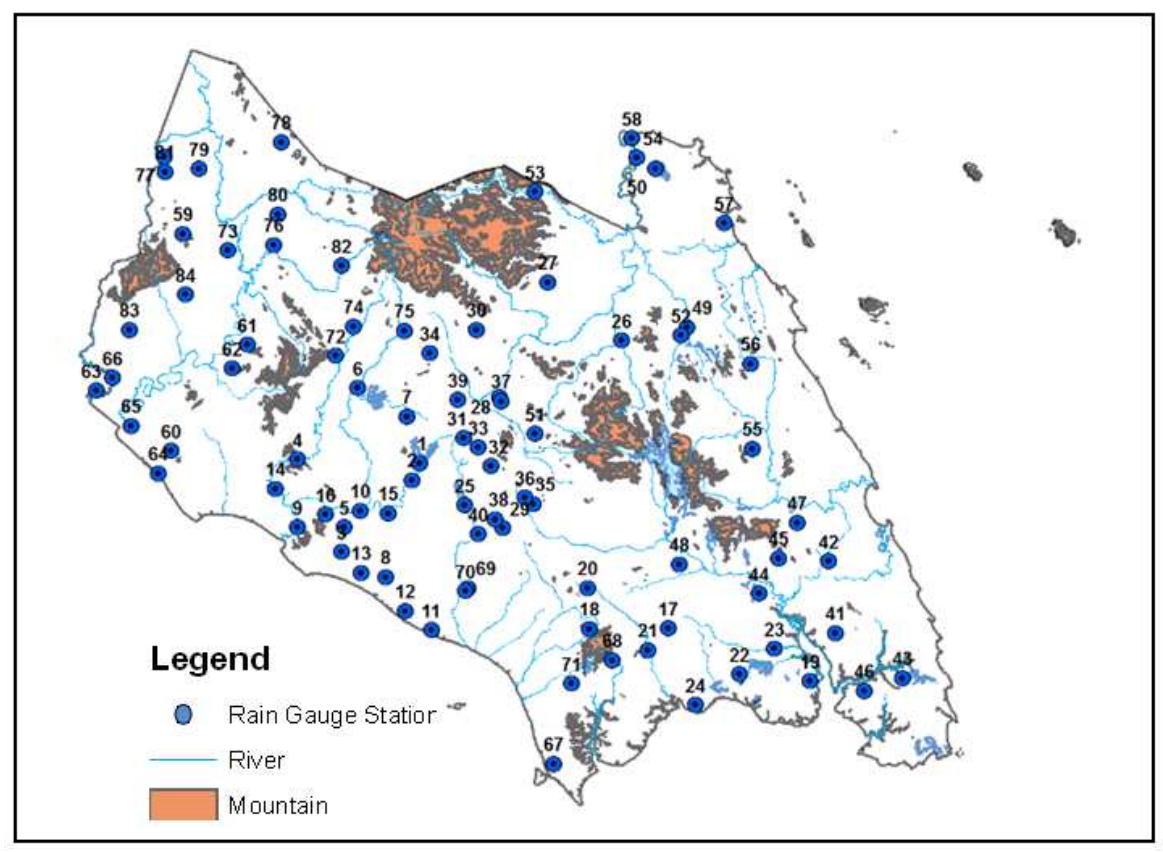

Figure 1: 84 Rain Gauge Locations in Johor

In Figure 2, the spatial autocorrelation of rainfall in Johor was examined with the semivariogram cloud. Different pairs of sample locations were examined by measuring the distance between two locations and the semivariogram was created by plotting half the difference squared between the values at the locations. Each dot in the semivariogram cloud represents a pair of location since locations that are close to each other should be more alike.

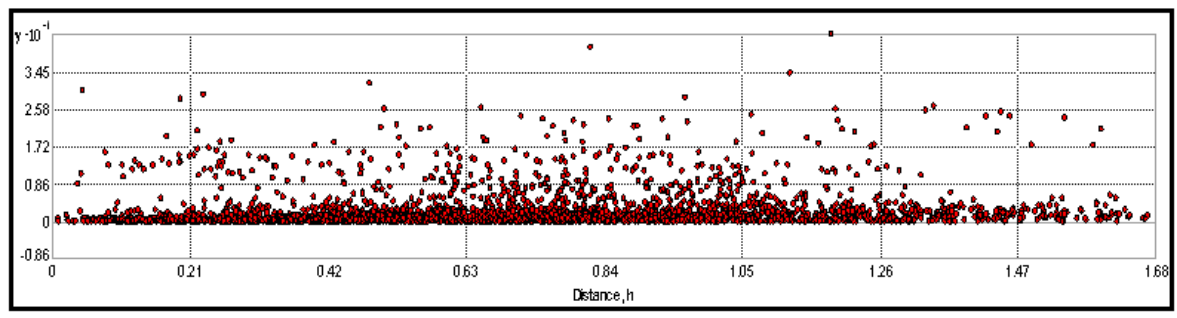

Figure 2: Semivariogram Cloud 


\section{Methodology}

This study used the combination of geostatistics method (variance-reduction method) and simulated annealing as the algorithm of optimization during the redesigned proses. Geostatistical analytic approach in this study is mainly based on two different operative steps:

1. Semivariogram Modelling

2. Optimization by Simulated Annealing.

\subsection{Semivariogram Modelling}

Kriging is a form of generalized linear regression for the formulation of an optimal estimator in a minimum mean square error sense [16]. The estimation variance $\sigma^{2}$ is a basic tool of variance reduction techniques for optimal selection of sampling locations. For the application of the variance reduction method to optimal location of sampling sites, a semivariogram must be modelled.

A semivariogram, $\gamma(h)$ is one of the significant functions to indicate spatial correlation in observations measured at sample locations. Semivariogram is represented as a graph that shows the difference in measure with distance between all pairs of sampled locations. Three common models for fitting semivariogram are the spherical, Gaussian and exponential models. A Gaussian model exhibits quadratic behavior near origin and produces short range correlations that are higher than for any second order stationary models with same range. Meanwhile, a spherical model shows a progressive decrease of spatial dependence until some distance, beyond which spatial dependence levels off. While an exponential model exhibits a less gradual pattern than a spherical model; spatial dependence decreases exponentially with increasing distance and disappears completely at an infinite distance [17].

There are three important elements in a fitted semivariogram: nugget, range and sill. The height that the semivariogram levelling takes off is called the sill, comprises of two parts: called the nugget the partial sill. The distance at which the semivariogram levelling takes place to the sill is called the range. The nugget effect represents the measurement errors or micro scale variations.

The estimated variance depends on the semivariogram model, the number $N$ of rain gauges and its spatial location. Therefore, choosing an appropriate semivariogram model is vital in determined the optimal estimation variance.

Let $h$ be the lag or distance, and $Z$ be an intrinsic random function and let $Z\left(x_{i}\right)$, for $i=1,2, \ldots, N$ be a sampling of size $N$. Then the following expression is an unbiased estimator for the semivariogram of the random function:

$$
\gamma(h)=\frac{1}{2 n(h)} \sum_{i=1}^{n(h)}\left[Z\left(x_{i}+h\right)-Z\left(x_{i}\right)\right]^{2}
$$

Equation (1) is used to compute experimental semivariogram from the data under study. By changing $h$, both in distance and direction, a set of the sample (or experimental) semivariograms for the data is obtained [18]. 
Three types of semivariogram models, Spherical, Gaussian and Exponential are selected to fit the data. The semivariogram models equation are as follow:

$$
\begin{aligned}
& \text { Spherical : } \gamma(h)=C\left(\frac{3}{2} \frac{h}{a}-\frac{1}{2}\left(\frac{h}{a}\right)^{3}\right) \\
& \text { Gaussian }: \gamma(h)=C\left(1-e^{-3\left(\frac{h}{a}\right)^{2}}\right) \\
& \text { Exponential }: \gamma(h)=C\left(1-e^{-\frac{3 h}{a}}\right)
\end{aligned}
$$

where $C$ is the sill and $a$ is the range.

The best model of semivariogram was selected based on the cross-validation statistics; mean error (ME), root mean square error (RMSE), average standardized error (ASE), mean standardized error (MSE) and root mean square standardized error (RMSS).

Once the model of the semivariogram is fixed, the estimation variance only depends on the number $N$ and the location of the rain gauges. To calculate the estimation variance using ordinary kriging,

$$
\sigma^{2}\left(x_{0}\right)=2 \sum_{i=1}^{k} \lambda_{i} \gamma\left(x_{i}, x_{0}\right)-\sum_{i=1}^{k} \sum_{j=1}^{k} \lambda_{i} \lambda_{j} \gamma\left(x_{i}, x_{j}\right)
$$

where

$$
\begin{aligned}
& \hat{Z}\left(x_{0}\right)=\sum_{i=1}^{k} \lambda_{i} Z\left(x_{i}\right) \\
& \text { Subject to } \sum_{i=1}^{k} \lambda_{i}=1 .
\end{aligned}
$$

This is an algorithm for the ordinary kriging estimation [16]:

1. Calculate each term in matrix $G$.

Let $x_{i}$ 's be the sampling sites of a sample subset of size $k, i=1,2, \ldots, k$ and let $\gamma\left(x_{i}, x_{j}\right)$ 's be the experimental variogram. Then the $G$ is the matrix

$$
G=\left[\begin{array}{lllll}
\gamma\left(x_{1}, x_{1}\right) & \gamma\left(x_{2}, x_{1}\right) & \ldots & \gamma\left(x_{k}, x_{1}\right) & 1 \\
\gamma\left(x_{1}, x_{2}\right) & \gamma\left(x_{2}, x_{2}\right) & \cdots & \gamma\left(x_{k}, x_{2}\right) & 1 \\
\cdots & \cdots & \cdots & \cdots & \cdots \\
\gamma\left(x_{1}, x_{k}\right) & \gamma\left(x_{2}, x_{k}\right) & \cdots & \gamma\left(x_{k}, x_{k}\right) & 1 \\
1 & 1 & \cdots & 1 & 0
\end{array}\right]
$$

2. Calculate each term in matrix $g$.

Let $x_{0}$ be the estimation location, then the $g$ is the matrix

$$
g=\left[\begin{array}{lllll}
\gamma\left(x_{0}, x_{1}\right) & \gamma\left(x_{0}, x_{2}\right) & \cdots & \gamma\left(x_{0}, x_{k}\right) & 1
\end{array}\right]^{\prime}
$$


3. Solve the system of equations

$$
\begin{gathered}
\qquad W=g \\
W=g G^{-1} \\
\text { Where } W=\left[\begin{array}{lllll}
\lambda_{1} & \lambda_{2} & \cdots & \lambda_{k} & -\mu
\end{array}\right]^{\prime} .
\end{gathered}
$$

4. Calculate the ordinary kriging estimation variance

$$
\sigma^{2}\left(x_{0}\right)=g^{\prime} W=g^{\prime} G^{-1} g
$$

\subsection{Optimization by Simulated Annealing (SA)}

The minimisation of the objective function given in this study is done by simulated annealing. SA was first introduced by Kirkpatrick in 1983 [19]In the rain gauge network problem, the energy of the annealing process is given by the value of objective function and the temperature, $T$ is a global time-varying parameter that is adjusted empirically for a given data set. For the starting of the simulation, the temperature must be set at a high value to permit the probability of any configuration of rain gauges, and the cooling process is done following a precise annealing schedule.

The annealing process for optimal location of $M$ rain gauges may be simulated through the following steps:

1. The initial configuration of rain gauges is obtained by randomly select $1,2,3, \ldots, M, M+$ $1, M+2, \ldots, N$ rain gauges available (in the optimal subset selection problem).

2. An energy is defined as a measure of the difference between different configurations. The energy is given by the objective function (equation 5).

3. The initial temperature is determined as:

$$
T_{0}=1000 \sigma^{2}
$$

where $T_{0}$, is the initial temperature, $\sigma^{2}$ is the estimated variance of the experimental data. As mention earlier, the initial $T$ must be set at a very high value. With the selection of 1000, there is a guarantee that the initial temperature is higher than the difference in energy between any two configurations taken at random.

4. The initial configuration is perturbed by randomly selecting one from $N$ of the rain gauges, and the objective function is calculated.

5. For each value of constant $T$, a number of $100 N$ new configurations are tried. The simulation remains at constant temperature until $100 N$ configurations have been tried and the minimum value of objective function for the configurations is accepted. 
6. For each new configuration, the algorithm must decide whether to reject it or accept it. Let $\triangle O F=O F_{\text {new }}-O F_{\text {old }}$. If $\triangle O F \leq 0$, the new configuration is accepted because the objective function has been minimized. However, if $\triangle O F \geq 0$, the new configuration is accepted with probability acceptation criterion, $e^{\frac{-\Delta O F}{T}}[6]$.

7. The temperature is decreased at a certain amount, in this study by $10 \%$ and step 5 is applied again.

8. The running of the simulation process is defined by steps 6 and 7 continues until:

i. a number of prefixed numbers of iterations is reached

ii. at a given constant $T$ none of the numbers of new configurations have been accepted

iii. changes in the objective function for various consecutive $T$ steps are slight.

\section{Results and Analysis}

\subsection{Semivariogram Modelling}

The first step in ordinary kriging is to construct a semivariogram from the data. A semivariogram consists of two parts: an experimental semivariogram and a model semivariogram. The experimental variogram is found by calculating the variance of each point in the set with respect to each of the other points and plotting the variances versus distance between the points (equation (1)). Once the experimental variogram is computed, the next step is to define a model variogram. A model variogram is a simple mathematical function that models the trend in the experimental variogram. The resulting of the three different semivariogram models is shown in Figure 4.

Table 1: Comparison of the Spherical, Exponential and Gaussian Semivariogram Models

\begin{tabular}{|c|c|c|c|}
\hline & Spherical & Exponential & Gaussian \\
\hline Nugget & 1.0783 & 0.93917 & 1.1962 \\
Sill & 1.498 & 1.5568 & 1.7059 \\
Range & 1.04903 & 1.05449 & 1.01199 \\
\hline
\end{tabular}

Figure 3 shows the three semivariogram models which are Gaussian, Spherical and Exponential. The semivariograms models were fitted to the empirical data using the equation (2-4). The spherical model shows a progressive decrease of spatial dependence until some distance, beyond which spatial dependence levels off. The exponential model meanwhile, exhibits a less gradual pattern than a spherical model; spatial dependence decreases exponentially with increasing distance and disappears completely at an infinite distance. Table 1 shows the comparison between each semivariogram model by comparing the nugget, partial sill and range value. Further analysis to decide which model is the most accurate model is done by cross validation scheme.

Cross validation technique is applied to compare the difference between the semivariogram models in order to validate the accuracy of each model. Cross validation is a reliable scheme 


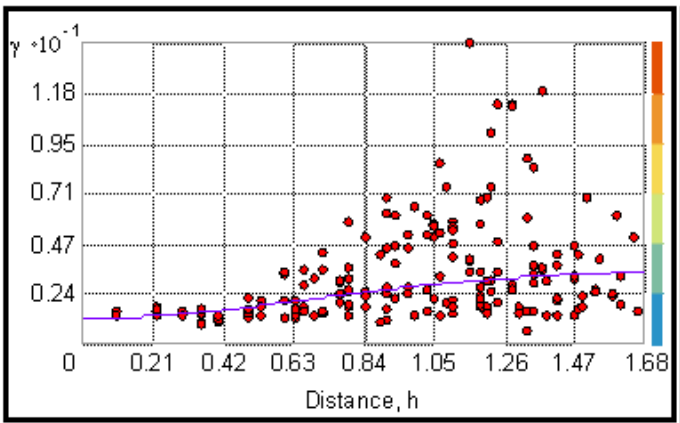

(a)

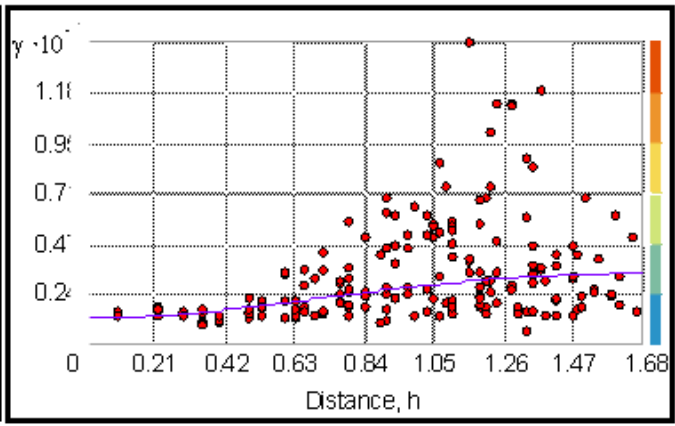

(b)



(c)

Figure 3: Three Types of Semivariogram Models Fitted the Data, (a) Gaussian, (b) Spherical and (c) Exponential.

to be used to determine the best semivariogram model among the available models. In cross validation technique, five different errors are calculated which are mean error (ME), root mean square error (RMSE), average standardized error (ASE), mean standardized error (MSE) and root mean square standardized error (RMSS).

$$
\begin{aligned}
\mathrm{ME} & =\frac{1}{n} \sum_{i=N}^{N}\left[z\left(x_{i}\right)-\hat{z}\left(x_{i}\right)\right] \\
\mathrm{RMSE} & =\sqrt{\frac{1}{n} \sum_{i=1}^{N}\left[z\left(x_{i}\right)-\hat{z}\left(x_{i}\right)\right]^{2}} \\
\mathrm{ASE} & =\sqrt{\frac{1}{n} \sum_{i=1}^{N} \sigma^{2}\left(x_{i}\right)} \\
\mathrm{MSE} & =\frac{1}{n} \sum_{i=1}^{N} \frac{M E}{\sigma^{2}\left(x_{i}\right)} \\
\mathrm{RMSS} & =\sqrt{\frac{1}{n} \sum_{i=1}^{N}\left(\frac{M E}{\sigma^{2}\left(x_{i}\right)}\right)^{2}}
\end{aligned}
$$

where $z\left(x_{i}\right)$ is the observed value, $\hat{z}\left(x_{i}\right)$ is the predicted value, $N$ is the number of values in the dataset and $\sigma^{2}$ is the kriging variance for location $x_{i}[20]$. 
In order to examine the quality of the three semivariogram models and determine the best fitted model, the error of each semivariogram is calculated. For a model that provides accurate predictions, the mean error value should be close to 0. Mean error is the average difference between the measured and the predicted values. Meanwhile, the root mean square (RMS) error is based on the square error and its value should be closer to 1 . The average standard error value should be near RMS error and the mean standardized error value should be near 0 . The RMS standardized error is the average standard error divided by the RMS and the value should be closer to 1 [21]. If it is greater than 1, then the prediction model underestimates the variability of the dataset but if it is less than 1, then the prediction model overestimates the variability of the dataset.

Table 2: Errors for Spherical, Exponential and Gaussian Model

\begin{tabular}{|c|c|c|c|}
\hline Errors & Spherical & Exponential & Gaussian \\
\hline Mean (ME) & 0.007532 & 0.007185 & 0.006474 \\
Root mean square (RMSE) & 1.402 & 1.392 & 1.428 \\
Average standard (ASE) & 1.154 & 1.185 & 1.155 \\
Mean standardized (MSE) & 0.005717 & 0.005195 & 0.004454 \\
RMS standardized(RMSSE) & 1.201 & 1.168 & 1.216 \\
\hline
\end{tabular}

Based on Table 2, it can be seen that the Gaussian model has the lowest mean error which is 0.006474 . The Gaussian model also has the nearest mean standardized value to 0 which is 0.004454. However, exponential semivariogram has the smallest value close to 1 for its RMS error with a value of 1.392. Since the average standard error value should be near RMS value, the exponential also has the lowest value different which is 0.207 . Other than that, the lowest RMS standardized value is also from the exponential model which is 1.168. This shows that exponential semivariogram is the best fitted experimental semivariogram for the study.

\subsection{Optimization by Simulated Annealin}

When the semivariogram is successfully fitted to the empirical rainfall data, the simulated annealing optimization method is applied to find the minimum objective function (equation 5 ) in order to get the optimum number and location of rain gauge stations. The optimization technique done based on the steps mentioned earlier. The results of the annealing process were shown in figure 5 below.

Table 3: Simulated Annealing Results

\begin{tabular}{|c|c|c|c|}
\hline & Spherical & Exponential & Gaussian \\
\hline Stations remained & 70 & 64 & 32 \\
Stations removed & 14 & 20 & 52 \\
\hline
\end{tabular}




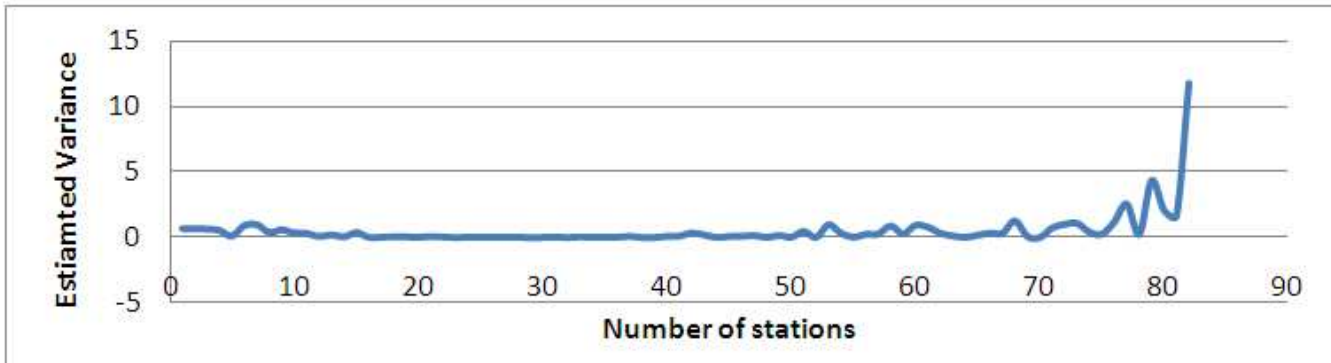

(a)

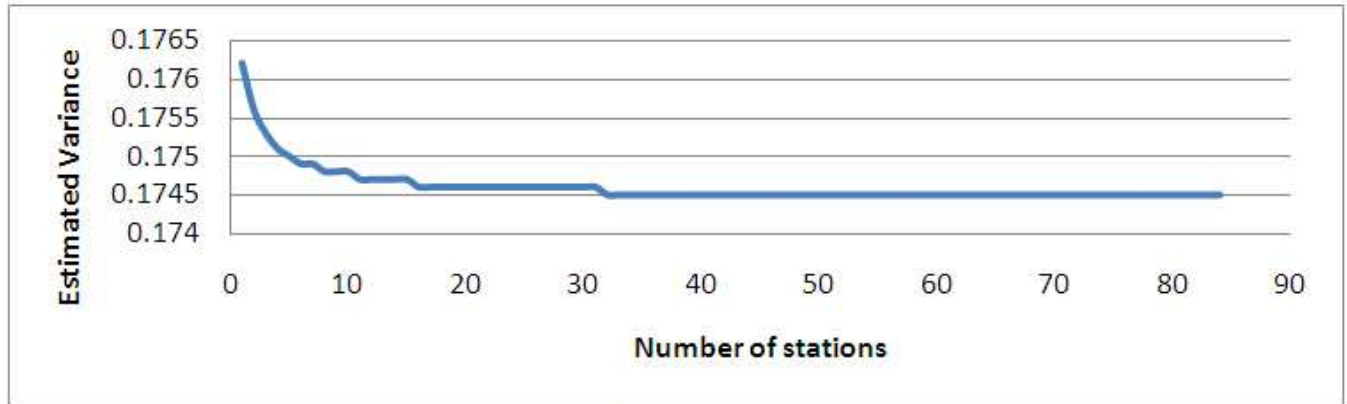

(b)

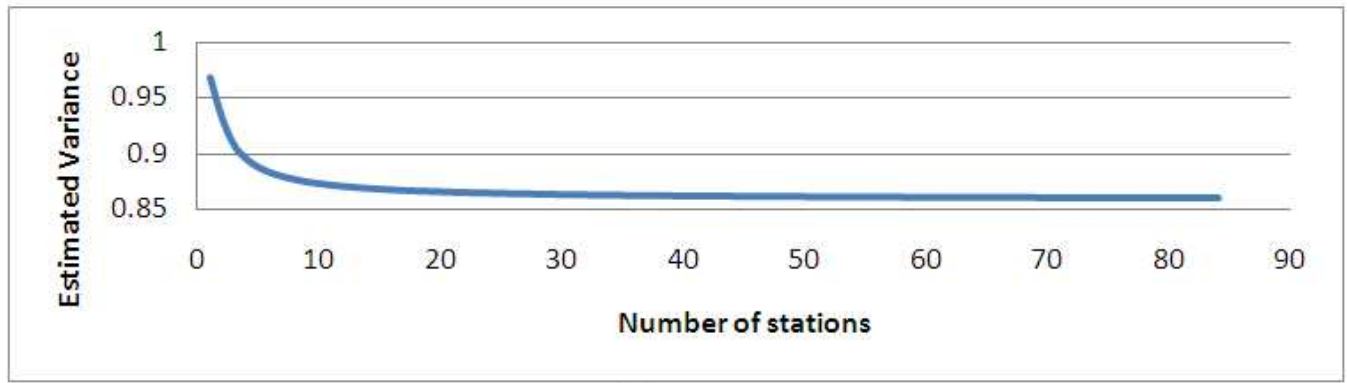

(c)

Figure 4: Estimation Variance Versus Number of Stations for (a) Spherical, (b) Gaussian and (c) Exponential Semivariogram

Figure 4 shows the results of the simulated annealing optimization process in determining the optimal number of rain gauge stations in Johor for three different semivariograms. The number of stations which are remained and removed is as shown in Table 3. Spherical model shows that 70 stations remained and 14 stations are removed. Meanwhile, the exponential depicts 64 remained stations and 20 removed stations. The Gaussian model on the other hand shows that 32 stations are remained and 52 stations need to be removed or relocated. The location of each station is shown as in Figure 5.

\section{Conclusion}

This study has shown that semivariogram plays an important role in variance reduction method. Three different semivariogram give different results in determining the optimal number and locations of rain gauge stations. From the cross validation scheme, exponential semi variogram 




Exponential

Figure 5: The Locations of the Remained and Removed Stations in Johor for (a) Spherical, (b) Gaussian and (c) Exponential

model is the best fitted model compared to the Spherical and Gaussian model based on the calculated ME, RMSE, ASE, MSE and RMSS. The results show that the errors fulfill the characteristics needed to be considered as an excellent semivariogram model.

Overall, this study has illustrated that the geostatistics method with simulated annealing technique can be used as the optimization method to provide the solution for optimal number and the location of the rain gauges in Johor state area in order to get better rainfall data. This model and methodology can provide information that will help decision makers to understand the relationship between numbers and locations of rain gauge stations in order to provide a better and more accurate rainfall data. Further researches on the optimization technique are required for better results.

\section{Acknowledgment}

The authors would like to thank the Universiti Malaysia Pahang under UMP grant RDU1703196 for their financial funding and support. 


\section{References}

[1] Bras, R. L. and Rodríguez-Iturbe, I. Network design for the estimation of areal mean of rainfall events. Water Resources Research. 1976. 12(6): 1185-1195.

[2] Bastin, G., Lorent, B., Duque, C. and Gevers, M. Optimal estimation of the average areal rainfall and optimal selection of rain gauge locations. Water Resources Research. 1984. 20(4): 463-470.

[3] Shamsi, U. M., Quimpo, R. G. and Yoganarasimhan, G. N. An application of kriging to rainfall network design. Hydrology Research. 1988. 19(3): 137-152.

[4] Kassim, A. H. M. and Kottegoda, N. T. Rainfall network design through comparative kriging methods. Hydrological Sciences Journal. 1991. 36(3): 223-240.

[5] Loof, R., Hjorth, P. and Raut, O. B. Rainfall network design using the kriging technique: A case study of Karnali river basin, Nepal. International Journal of Water Resources Development. 1994. 10(4): 497-513.

[6] Pardo-Igúzquiza, E. Optimal selection of number and location of rainfall gauges for areal rainfall estimation using geostatistics and simulated annealing. Journal of Hydrology. 1998. 210(1-4): 206-220.

[7] Chen, Y. C., Wei, C. and Yeh, H. C. Rainfall network design using kriging and entropy. Hydrological Processes: An International Journal. 2008. 22(3): 340-346.

[8] Cudennec, C., El Kamel, H. F. and Slimani, M. A comparison of three geostatistical procedures for rainfall network optimization. International Renewable Energy Congress. 2010. 8.

[9] Chebbi, A., Bargaoui, Z. K. and Cunha, M. D. C. Optimal extension of rain gauge monitoring network for rainfall intensity and erosivity index interpolation. Journal of Hydrologic Engineering. 2011. 16(8): 665-676.

[10] Awadallah, A. G. Selecting optimum locations of rainfall stations using kriging and entropy. International Journal of Civil and Environmental Engineering IJCEE-IJENS. 2012. 12(1): 36-41.

[11] Al-Aboodi, A. H. D. and Al-Abadi, A. M. Optimum rain-Gauges network design of some cities in Iraq. Journal of University of Babylon. 2014. 22(4): 938-950.

[12] Adhikary, S. K., Yilmaz, A. G. and Muttil, N. Optimal design of rain gauge network in the Middle Yarra River catchment, Australia. Hydrological processes. 2015. 29(11): 2582-2599.

[13] Bohling, G. C. Introduction to geostatistics. Kansas Geological Survey Open File Report no. 26(2007): 50 .

[14] Mazzella, A. and Mazzella, A. The importance of the model choice for experimental semivariogram modeling and its consequence in evaluation process. Journal of Engineering. 2013.

[15] Guide to Meteorological Instruments and Methods of Observation. WMO-No.8. 7th Edition, WMO, Geneva. 2008.

[16] Olea, R. A. Geostatistics for Engineers and Earth Scientists. Springer Science and Business Media. 2012. 
[17] Chang, K. T. Introduction to Geographic Information Systems. Boston: McGraw-Hill Higher Education. 2006. 117-122.

[18] Burrough, P. A. Principles of Geographical Information Systems for Land Resources Assessment. 1986.

[19] Kirkpatrick, S., Gelatt, C. D. and Vecchi, M. P. Optimization by simulated annealing. Science. 1983. 220(4598): 671-680.

[20] Robinson, T. P. and Metternicht, G. Testing the performance of spatial interpolation techniques for mapping soil properties. Computers and Electronics in Agriculture. 2006. 50(2): 97-108.

[21] ESRI, ArcGIS 9 Using ArcGIS Spatial Analyst, Retrieved from http://dusk2.geo.orst.edu/gis/geostat_analyst.pdf, 2001. 